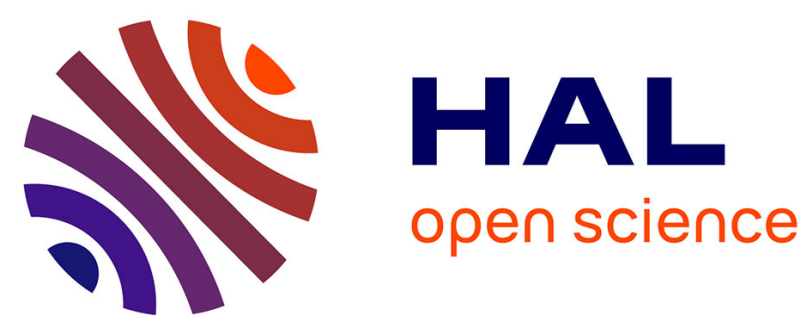

\title{
Effect of surface polishing and oxidization induced strain on electronic order at the Verwey transition in Fe3O4
}

Wojciech Tabis, Emilio Lorenzo, A. Kozlowski, T. Kolodziej, Z. Tarnawski, Z. Kakol, C. Mazzoli, H. Walker, N. Jaouen, Danny Mannix, et al.

\section{- To cite this version:}

Wojciech Tabis, Emilio Lorenzo, A. Kozlowski, T. Kolodziej, Z. Tarnawski, et al.. Effect of surface polishing and oxidization induced strain on electronic order at the Verwey transition in Fe3O4. Journal of Physics: Condensed Matter, 2013, 25 (5), pp.055603. 10.1088/0953-8984/25/5/055603 . hal00976917

\section{HAL Id: hal-00976917 https://hal.science/hal-00976917}

Submitted on 10 Apr 2014

HAL is a multi-disciplinary open access archive for the deposit and dissemination of scientific research documents, whether they are published or not. The documents may come from teaching and research institutions in France or abroad, or from public or private research centers.
L'archive ouverte pluridisciplinaire HAL, est destinée au dépôt et à la diffusion de documents scientifiques de niveau recherche, publiés ou non, émanant des établissements d'enseignement et de recherche français ou étrangers, des laboratoires publics ou privés. 
Effect of surface polishing and oxidization induced strain on electronic order at the Verwey transition in $\mathrm{Fe}_{3} \mathrm{O}_{4}$

This article has been downloaded from IOPscience. Please scroll down to see the full text article.

2013 J. Phys.: Condens. Matter 25055603

(http://iopscience.iop.org/0953-8984/25/5/055603)

View the table of contents for this issue, or go to the journal homepage for more

Download details:

IP Address: 147.173.59.86

The article was downloaded on 13/02/2013 at 17:48

Please note that terms and conditions apply. 


\title{
Effect of surface polishing and oxidization induced strain on electronic order at the Verwey transition in $\mathrm{Fe}_{3} \mathrm{O}_{4}$
}

\author{
W Tabis ${ }^{1,2}$, J E Lorenzo ${ }^{3}$, A Kozlowski ${ }^{1}$, T Kolodziej $^{1}$, Z Tarnawski $^{1}$,

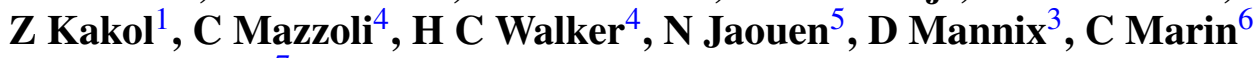 \\ and $\mathbf{J}$ M Honig ${ }^{7}$ \\ ${ }^{1}$ AGH University of Science and Technology, Faculty of Physics and Applied Computer Science, \\ Al. A. Mickiewicza 30, 30-059 Krakow, Poland \\ ${ }^{2}$ School of Physics and Astronomy, University of Minnesota, Minneapolis, MN 55455, USA \\ ${ }^{3}$ Institut Néel-CNRS/UJF, F-38042 Grenoble, Cedex 9, France \\ ${ }^{4}$ European Synchrotron Radiation Facility, F-38054 Grenoble Cedex 9, France \\ ${ }^{5}$ Synchrotron SOLEIL, L'Orme des Merisiers Saint-Aubin, BP 48 F-91192, Gif-sur-Yvette Cedex, \\ France \\ ${ }^{6}$ SPSMS, UMR-E 9001, CEA-INAC/UJF-Grenoble 1, IMAPEC, F-38054 Grenoble Cedex 9, France \\ ${ }^{7}$ Department of Chemistry, Purdue University, West Lafayette, IN 47907, USA
}

E-mail: tabis@physics.umn.edu and emilio.lorenzo@grenoble.cnrs.fr

Received 27 September 2012, in final form 24 November 2012

Published 8 January 2013

Online at stacks.iop.org/JPhysCM/25/055603

\begin{abstract}
Following the controversy between two previous publications (Lorenzo et al 2008 Phys. Rev. Lett. 101226401 and Garcia et al 2009 Phys. Rev. Lett. 102 176405), we report on the influence of mechanical polishing, and subsequent sample storage, on the electronic order at the Verwey transition of highly pure magnetite, $\mathrm{Fe}_{3} \mathrm{O}_{4}$, by resonant x-ray scattering. Contrary to expectations, mechanically polishing the surface induces an inhomogeneous micron deep dead layer, probably of powdered $\mathrm{Fe}_{3} \mathrm{O}_{4}$. In addition, we have found that polishing the sample immediately before the experiment influences and favors the appearance of long range order electronic correlations, whereas samples polished well in advance have their electronic order quenched. Conversely, lattice distortions associated with the Verwey transition appear less affected by the surface state. We conclude that mechanical polishing induces stresses at the surface that may propagate into the core of the single crystal sample. These strains relax with time, which affects the different order parameters, as measured by $\mathrm{x}$-ray resonant diffraction.
\end{abstract}

(Some figures may appear in colour only in the online journal)

\section{Introduction}

Charge and orbital order play an important role in the still not fully explained metal-insulator transition in magnetite, $\mathrm{Fe}_{3} \mathrm{O}_{4}$. Magnetite is regarded as one of the canonical prototype Mott insulators and the interplay among the charge, orbital and lattice degrees of freedom is particularly vital in understanding this class of compounds [1]. The occurrence and the relationship between the different electronic orders, charge (CO) and orbitals (OO), and the lattice distortions
(LD) originating in the neighborhood of the Verwey transition temperature, $T_{\mathrm{V}} \approx 123 \mathrm{~K}$, in magnetite has been the subject of intense studies in the recent years. The role of charge order at the transition has been emphasized in a number of studies [2, 3], starting from the original idea of Verwey [4]. After some contradictory studies and results during the last 10 years, the consensus to which the scientific community has adhered to is that there is a slight charge order developing below $T_{\mathrm{V}}$ at the Fe-octahedral sites, the charge amounting to $2.5 \pm \delta$, and $\delta=0.12 e$ [5]. The metal-insulator transition takes place by 
capturing and binding an extra electron in the fully occupied, quasi-degenerated $t_{2 g}$ orbitals. As a result, 3-band electron calculations [6] predict a very complex phase diagram of real and complex orbital orders and $a b$ initio band structure calculations [7-10] point out the occurrence of an electronic orbital order in the low temperature phase of magnetite. Resonant $\mathrm{x}$-ray scattering (RXS) experiments at the $\mathrm{Fe}$ $\mathrm{L}_{2,3}$-edges [11] have identified the peak $(001 / 2)_{\mathrm{C}}$ (in the notation of the cubic unit cell) as due to OO, although doubts have been cast on the validity of this interpretation [12].

An important issue in the science of correlated electron systems is the nature and interplay of the electronic order(s). RXS studies at the $\mathrm{Fe} \mathrm{K}$-edge of $\mathrm{Fe}_{3} \mathrm{O}_{4}$ have revealed the surprising feature of the different behavior of these three orderings [13] at $T_{\mathrm{V}}$. This is indicated by their different temperature dependence and in particular by the absence of a jump of the scattered intensity right at $T_{\mathrm{V}}$ for $\mathrm{CO}$ and $\mathrm{OO}$ reflections whereas those related to the pure LD do display this characteristic feature. Unlike the monoclinic LD, that disappears abruptly at $T_{\mathrm{V}}, \mathrm{CO}$ and $\mathrm{OO}$ long range order fluctuations survive the transition and smoothly disappear at temperatures as high as $10 \mathrm{~K}$ above $T_{\mathrm{V}}$ [13]. However, it is not only the temperature variation of the intensity, the correlation length of the CO fluctuations displays a kink at this temperature, as well. At that time we associated this temperature with the location of the isotropy point seen in magnetization studies and it seemed plausible that electronic order may lead to a magnetic moment reorientation. These results were questioned in an analogous RXS study [14] where an abrupt disappearance of all three orderings LD, CO and OO at $T_{\mathrm{V}}$ was observed. Therefore this controversy on the role and nature of the electronic orders in magnetite at the Verwey transition still remains open.

Here, we present a new RXS study of the charge and orbital orders and of the lattice distortion associated with the cubic $(F d \overline{3} m)$ into monoclinic $(C c)$ transformation at the Verwey transition. The aim of the present work is to provide a unified description of the results shown in the previous papers $[13,14]$. To that end we have reproduced the experimental data shown in these two publications by applying different experimental protocols to the same high quality single crystal of stoichiometric magnetite and to Zn-doped and non-stoichiometric magnetite samples.

\section{Experiment}

RXS experiments were carried out at ID20 beamline of the ESRF in Grenoble, France [15]. A Si(111) double crystal monochromator, with an energy resolution of $0.8 \mathrm{eV}$, was used to select the $\mathrm{x}$-rays of the required energy in the neighborhood of the $\mathrm{Fe} \mathrm{K}$-edge in $\mathrm{Fe}_{3} \mathrm{O}_{4}(E=7.119 \mathrm{keV})$. The incident photon beam was linearly polarized in the horizontal direction and the scattering plane was parallel to the polarization vector ( $\sigma$-polarization). The scattered beam polarization was analyzed by means of a $\mathrm{MgO}(222)$ analyzer crystal in both $\sigma$ and $\pi$-polarizations.

The electronic orders in magnetite have been studied at the $\mathrm{Fe}$ K-edge $(7.119 \mathrm{keV})$. The $(001)_{\mathrm{C}}$ reflection is known to display the spectroscopic features of the charge order [13]. It has been measured in the $(\sigma-\sigma)$ polarization channel. The $(007 / 2)_{\mathrm{C}}$ is an extinct Bragg reflection (presumably related to the orbital order) that was studied in the $\sigma-\pi$ channel [16]. The $(003)_{\mathrm{C}}$ lattice distortion peak has been studied at an energy well below the edge $(7.05 \mathrm{keV})$ in the $\sigma-\sigma$ polarization channel. The sample temperature was controlled by a standard displex cryostat and the $\theta-2 \theta$ scans of the examined reflections were performed using a $4+1$ circle diffractometer in the vertical scattering geometry. All the samples were slowly ramped up in temperature from $90 \mathrm{~K}$. A single reflection was followed at a time and then the sample cooled down again.

One feature that we found of paramount importance in our RXS studies on $\mathrm{Fe}_{3} \mathrm{O}_{4}$ was the use of mechanical surface polishing either right before the experiment or well in advance, as has been reflected by the discrepancies between previous works $[13,14]$. The temperature evolution at resonance of the $(001)_{\mathrm{C}}$ and $(007 / 2)_{\mathrm{C}}$ reflections in the high quality single crystal of stoichiometric magnetite, $T_{\mathrm{V}}=124 \mathrm{~K}$, was studied twice. The first run type (we call it run A) was performed with the sample surface polished in advance, typically a couple of months before the experiment and stored in a desiccator under vacuum of the order of 10 Torr. The same sample was studied a second time, but with its surface polished and immediately inserted into the cryostat. This is the run type B. Samples were polished with sand paper and then $6 \mu \mathrm{m}$ diameter diamond paste. Interestingly these cycles have been repeated several times on the same sample and the results were alike. Subsequent diffraction experiments where the surface was polished with paste of smaller grain size $(0.2 \mu \mathrm{m})$ have also been carried out and confirmed the previous findings.

Polishing the surface of a crystal prior to measurements is typically used to (i) remove all traces of impurities (solvents, oxides, etc) affecting the surface of the sample. This is important in specific surface sensitive experiments like photoemission, tunneling microscopy, etc (ii) to gently remove the first microns of the sample after having been cut and thus eliminate the powdered sample that may appear at the surface. Or (iii) to remove scratches, deformations and other defects that may hinder the reflectivity of the sample. In all cases it is assumed that the effect of polishing is favorable for the sample measurements and that no damage or change is induced as a consequence of this action. Another important feature that is worth recalling is that polishing should be followed by a thermal cycling of the sample. As this last part is time consuming and requires special furnaces it is often not carried out in RXS experiments. Recently it has been shown that a clean sample of magnetite, for soft x-ray standards, can be found by cleaving the sample prior to the measurements [17]. This is not the standard procedure in the medium x-ray range so we did not use this technique in our samples. 


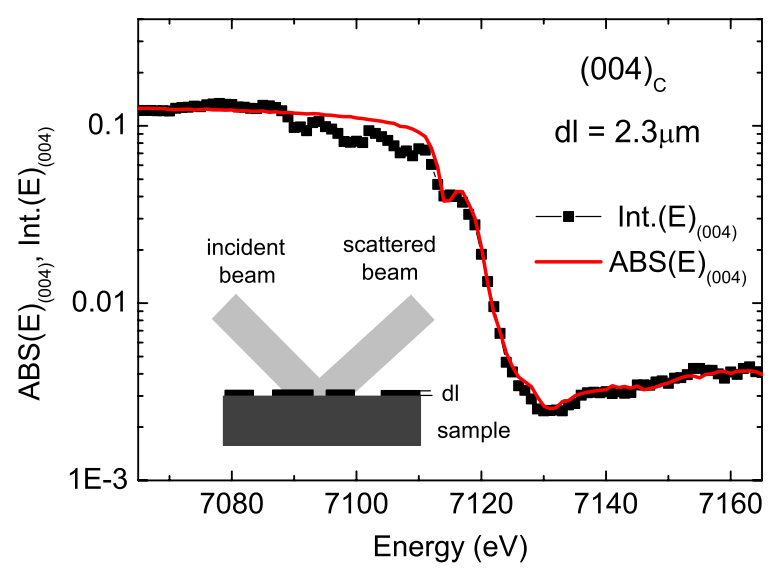

Figure 1. Black squares show the energy dependence of the $(004)_{C}$ peak intensity in run $\mathrm{A}$, at $50 \mathrm{~K}$. The solid line shows the $A B S(E)$ calculated by means of equation (2), with the linear absorption coefficient estimated from fluorescence data. The comparison yields $d_{\mathrm{d} l}=2.3 \mu \mathrm{m}$. The inset represents a sample with the dead layer providing a possible explanation for the intensity reduction found in the data.

\section{Dead layer estimation and the absorption correction}

If part of a sample contains chemical impurities or lattice imperfectness, this volume does not contribute to the coherent scattering in a diffraction experiment but only reduces the $\mathrm{x}$-ray beam during the absorption process. The diffracting part of the sample lies usually under the defected layer (called here as the dead layer), and thus the number of photons scattered is reduced as the incident and the diffracted beams are absorbed on passing through it. In this case we can treat the sample as a composition of two absorbers: the dead layer with thickness $d_{\mathrm{d} l}$ right underneath the surface, with the linear absorption coefficient $\mu_{\mathrm{d} l}(E)$, and the sample core with the linear absorption coefficient $\mu_{\mathrm{Fe}_{3} \mathrm{O}_{4}}(E)$. The total absorption coefficient $A B S_{(h k l)}(E)$ at reflections $(h k l)$ is then expressed as the absorption coefficient of the sample core $\mathrm{Fe}_{3} \mathrm{O}_{4}$ reduced by the absorption of the dead layer:

$$
\begin{aligned}
A B S_{(h k l)}(E)= & \frac{1}{g_{(h k l)} \mu_{\mathrm{Fe}_{3} \mathrm{O}_{4}}(E)}-\frac{1}{g_{(h k l)} \mu_{\mathrm{d} l}(E)} \\
& \times\left(1-\exp \left(-g_{(h k l)} \mu_{\mathrm{d} l}(E) d_{\mathrm{d} l}\right)\right) .
\end{aligned}
$$

Here, $g_{(h k l)}$ is the geometrical factor that accounts for the incident and scattered beams direction with respect to the surface of the sample. $g_{(h k l)}=\sin ^{-1}(\alpha)+\sin ^{-1}(\beta)$ with $\alpha(\beta)$ the angle between the incident (scattered) beam and the sample surface. As always in these calculations of Bragg scattering the thickness of the sample was set to infinity. If the chemical composition of the dead layer and the sample core are comparable, a very good approximation is $\mu_{\mathrm{Fe}_{3} \mathrm{O}_{4}}(E) \approx$ $\mu_{\mathrm{d} l}(E)=\mu(E)$, and equation (1) can be written as:

$$
A B S_{(h k l)}(E)=\frac{\exp \left(-g_{(h k l)} \mu(E) d_{\mathrm{d} l}\right)}{g_{(h k l)} \mu(E)} .
$$

The estimation of the thickness of the dead layer relies on an assumption that for any strong Bragg reflection (e.g.

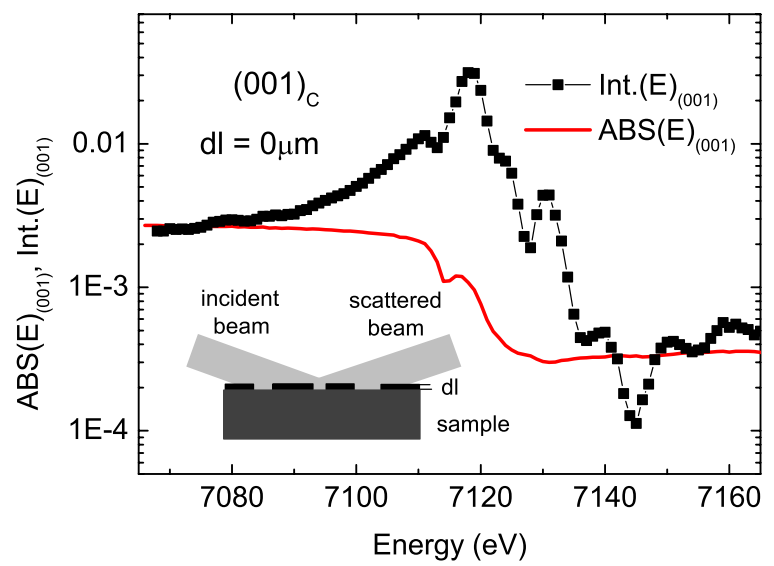

Figure 2. Energy dependence of the $(001)_{C}$ peak intensity in run $A$, at $50 \mathrm{~K}$, compared to the total absorption coefficient ABS(E) calculated by means of equation (2), with linear absorption coefficient estimated from fluorescence data. The experimental data shows an increase of the intensity at the resonance due to $\mathrm{CO}$. To match the step of $A B S_{(001)}(E)$ to that of the experimental data $d_{\mathrm{d} l}=0 \mu \mathrm{m}$ was taken for the calculations. The low diffraction angle for the $(001)_{C}$ reflection, shown in the inset, causes the $\mathrm{x}$-rays to shine on a larger area of the sample surface. For an inhomogeneous dead layer its effective thickness is thinner than in case of the $(004)_{\mathrm{C}}$.

$\left.(004)_{C}\right)$ the main contribution to its energy dependence is the absorption, and thus its intensity $\operatorname{Int}_{(h k l)}(E)$ is proportional to $A B S_{(h k l)}(E)$. Note that, $\log (\mu(E)) \ll \mu(E)$ and therefore the $\log \left(\operatorname{Int}_{(004)}(E)\right) \propto \mu(E)$.

In figure 1 , the energy dependence of the $(004)_{C}$ peak intensity is compared to the total absorption coefficient $A B S_{(004)}(E)$, where the linear absorption coefficient $\mu(E)$ was obtained from the fluorescence spectra. The comparison for this reflection yields a dead layer of $2.3 \mu \mathrm{m}$ and $1 \mu \mathrm{m}$ in runs $\mathrm{A}$ and $\mathrm{B}$, respectively. This is a very important result that deserves to be emphasized. Despite a continuous effort to create an appropriate surface by mechanical polishing an average micron deep dead layer is always found in our scans, in both run types A and B. Furthermore, the analysis of a number of strong reflections gives different effective dead layer for each peak (from $8 \mu \mathrm{m}$ for $(007)_{\mathrm{C}}$ to $1.8 \mu \mathrm{m}$ for $\left.(003)_{\mathrm{C}}\right)$ indicating that the dead layer is inhomogeneous throughout the surface of the sample. Our argument is additionally supported by the analysis of the low-angle reflections, such as $(001)_{\mathrm{C}}$, which shows a negligibly thin dead layer (see figure 2). In this case the $\mathrm{x}$-ray beam impinges the surface at an angle of $\approx 5^{\circ}$ and hence a large portion of the surface is illuminated (see insets in figures 1 and 2 ). In the scenario of inhomogeneous dead layer, a small, undefected part of the sample, that is directly exposed to $\mathrm{x}$-rays, will have larger scattering power than a large part buried under a thick dead layer. With decreasing scattering angle, and thus increasing the beam spot on the sample, the scattered intensity increases. A second result of figure 1 is that the energy dependence of the $(004)_{C}$ reflection intensity calculated according to equation (2) gave a qualitatively very good fit to the experimental data and thus the assumption on which this equation is derived, $\mu_{\mathrm{d} l}(E) \equiv \mu_{\mathrm{Fe}_{3} \mathrm{O}_{4}}(E)=\mu(E)$, 


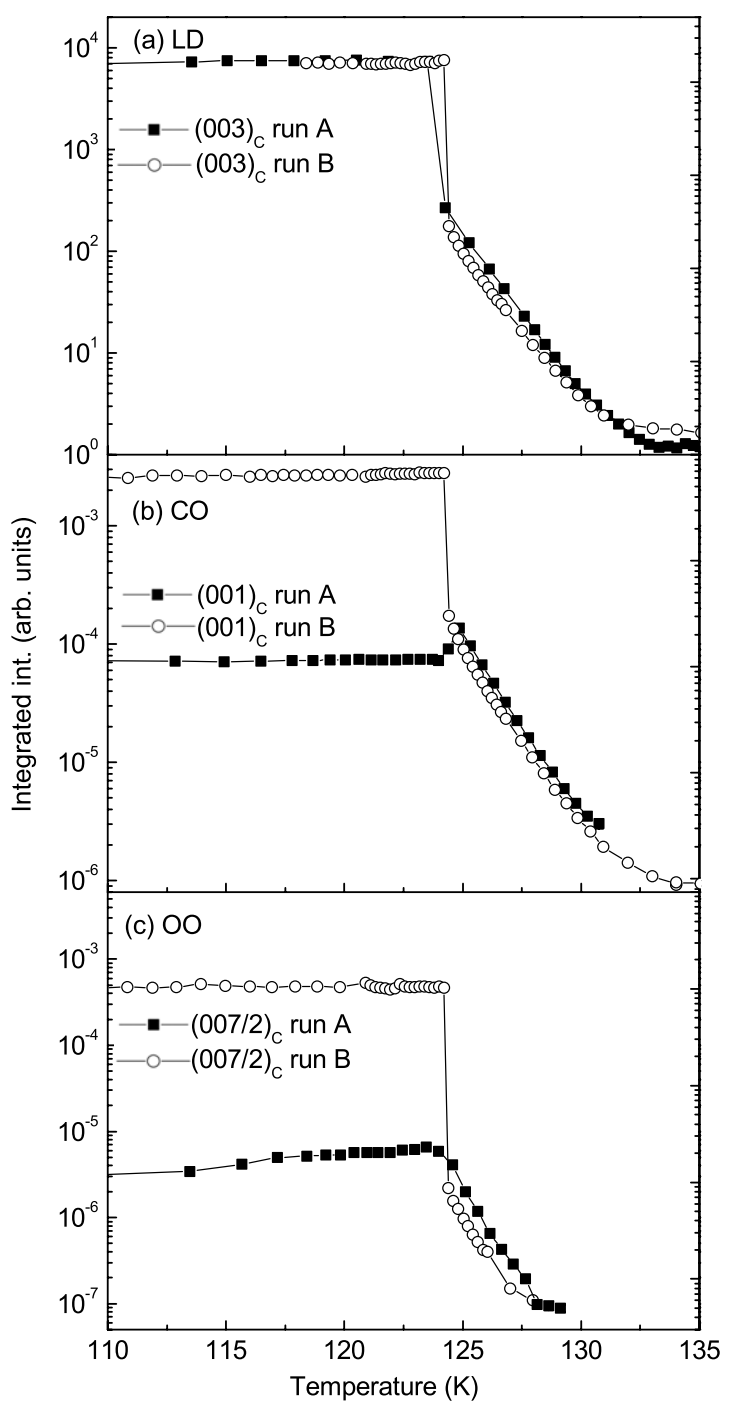

Figure 3. (a) Temperature evolution of the intensity of the $(003)_{\mathrm{C}}$ reflection of pure $\mathrm{Fe}_{3} \mathrm{O}_{4}$ for the surface polished well in advance before (squares) and immediately prior (circles) to the measurements. The photon energy was $7.05 \mathrm{keV}$, well below the resonance. (b) Idem for the $(001)_{\mathrm{C}}$ reflection. (c) Idem for the $(007 / 2)_{\mathrm{C}}$ reflection. The photon energy was $7.119 \mathrm{keV}$, right at the Fe K-edge resonance, for the two last reflections. The solid lines are guides to the eye.

holds to a large extent. Therefore we can conclude that the dead layer is made up of structurally damaged but chemically clean $\mathrm{Fe}_{3} \mathrm{O}_{4}$.

\section{Results}

\subsection{Stoichiometric $\mathrm{Fe}_{3} \mathrm{O}_{4}$}

Figures 3(a)-(c) shows the temperature evolution of the intensities of the $(003)_{\mathrm{C}},(001)_{\mathrm{C}}$ and $(007 / 2)_{\mathrm{C}}$ reflections, for runs A and B. The FWHM of the $(001)_{C}$ and $(007 / 2)_{C}$ reflections for both runs A and B is shown in figure 4 . The onset of the long range electronic correlations is invariably observed at temperatures $10 \mathrm{~K}$ above $T_{\mathrm{V}}$ in both $\mathrm{A}$ and $\mathrm{B}$ temperature runs as previously found [13]. However, the

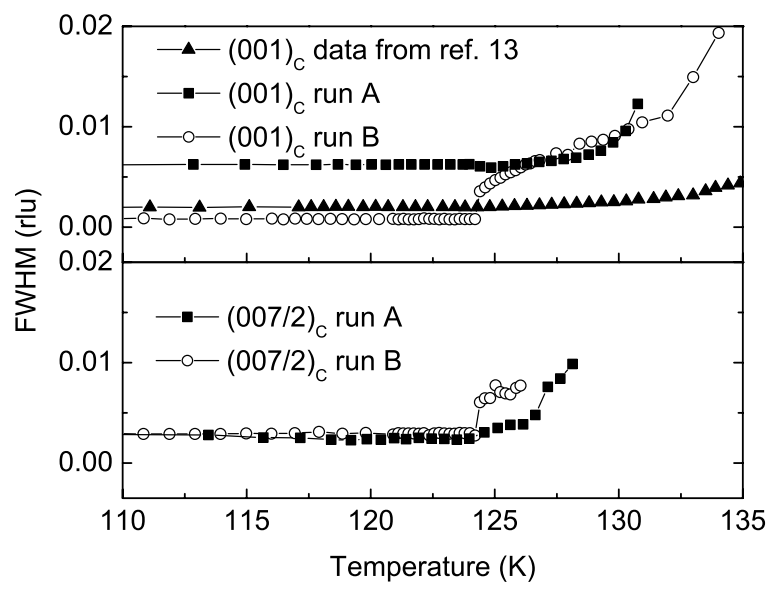

Figure 4. Temperature dependence of FWHM of the $(001)_{\mathrm{C}}$ and $(007 / 2)_{C}$ reflections for the surface polished well in advance (squares) and immediately prior (circles) to the measurements. The data from [13] (triangles), shown here for comparison, are shifted in order to compensate the difference in $T_{\mathrm{V}} \mathrm{s}$. The momentum scans has been performed along $q=(00 l)$. The solid lines are guides to the eye.

essential difference between the $\mathrm{A}$ and $\mathrm{B}$ runs arises at the structural transition temperature, where an additional expansion of the charge and orbital ordered regions in the $\mathrm{B}$ runs is observed as a sudden increase of the corresponding $(001)_{\mathrm{C}}$ and $(007 / 2)_{\mathrm{C}}$ peak intensities. Additionally, an abrupt increase of the correlation lengths of $\mathrm{CO}$ and $\mathrm{OO}$ at $T_{\mathrm{V}}$ is characteristic only for the B runs: this may be concluded from the temperature dependence analysis of FWHM of the $(001)_{\mathrm{C}}$ and the $(007 / 2)_{\mathrm{C}}$ peak that suddenly decrease at $T_{\mathrm{V}}$ on sample cooling across the transition (see figure 4). These results clearly indicate that the surface polishing/preparation has an impact on the intensity of the electronic orderings at $T_{\mathrm{V}}$, and thus on the size of the percolating regions.

These differences can explain the discrepancies between the results presented in the previous publications, which cannot just be due to the sample quality as pointed out in [14]. The magnitude of the intensity drop of the structural reflections across $T_{\mathrm{V}}$ indicates that the samples studied are of very high quality. The effect of a run B is more important for the $(007 / 2)_{C}$ reflection than for the $(001)_{C}$. As seen from figure 3 the intensity ratio (run B/run A) in the ordered phase (monoclinic) for the $(007 / 2)_{\mathrm{C}}$ reflection amounts to a factor of more than 150 whereas it is only a factor of 40 for the $(001)_{C}$. The intensity drop of the $(003)_{C}$ is essentially independent of the preparation procedure.

\subsection{Zn-doped and non-stoichiometric $\mathrm{Fe}_{3} \mathrm{O}_{4}$}

The influence of doping/non-stoichiometry on CO and LD in magnetite has also been investigated in two similar samples, polished well before the experiment (run A) $\mathrm{Fe}_{3-x} \mathrm{Zn}_{x} \mathrm{O}_{4}$, $x=0.0085$ and polished right before the experiment (run $\mathrm{B}$ ) $\mathrm{Fe}_{3(1-\delta)} \mathrm{O}_{4} \delta=0.0025$ (figure 5). Samples and concentrations were chosen such that the corresponding Verwey temperatures were as close as possible between them. The dependence of $T_{\mathrm{V}}$ on non-stoichiometry and $\mathrm{Zn}$ concentration can be 


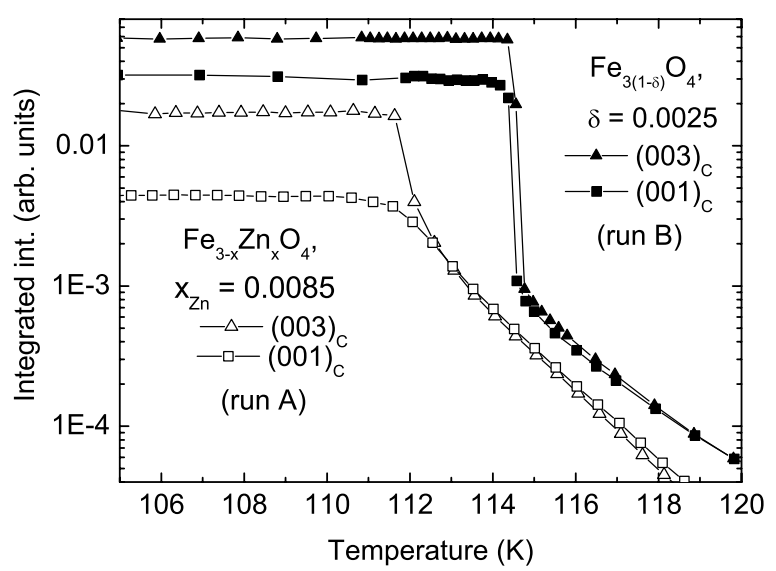

Figure 5. Comparison of the temperature dependence of the $(001)_{C}$ and $(003)_{C}$ peaks intensities for polished well before the experiment (run A) $\mathrm{Fe}_{3-x} \mathrm{Zn}_{x} \mathrm{O}_{4}, x=0.0085$ and polished right before the experiment (run $\mathrm{B}$ ) $\mathrm{Fe}_{3(1-\delta)} \mathrm{O}_{4} \delta=0.0025$ samples. The solid lines are guides to the eye.

found in the literature [18]. The detailed analysis of the temperature evolution of $\mathrm{CO}$ and LD (see figure 5), first, confirms the influence of the sample polishing right before the experiment on the charge ordering process and, second, proves that non-stoichiometry is not a parameter determining the amplitude of the step of the $(001)_{C}$ reflection intensity. Whereas the temperature evolution of the intensity of the

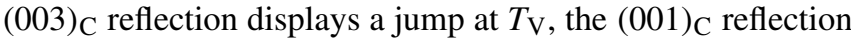
evolves just in the same way as found in the pure sample. Again, a smooth development of the $\mathrm{CO}$ above the Verwey transition $\left(T_{\mathrm{V}}=112 \mathrm{~K}\right)$ is observed for the $\mathrm{Zn}$-doped sample (run A). The step of the $(001)_{C}$ reflection intensity at the transition $\left(T_{\mathrm{V}}=114.5 \mathrm{~K}\right)$ for non-stoichiometric sample indicates an additional expansion of the charge ordered regions, characteristic for all run $\mathrm{B}$ samples.

The energy dependence of the $(001)_{C}$ peak intensity in pure $\mathrm{Fe}_{3} \mathrm{O}_{4}$ is shown in figure 6. Again, the same quantity was measured twice, in both A and B runs. The comparison of the results collected during the two runs shows no major differences suggesting that the spectroscopic features related to the $\mathrm{CO}$ itself are not affected by polishing. It can be thus concluded that only the development of the long range order at $T_{\mathrm{V}}$ is influenced by mechanical treatment of the surface and the polishing favors the sudden growth of the order. Since, $\mathrm{CO}$ and $\mathrm{OO}$ are observed at $\mathrm{Fe} \mathrm{K}$-edge where the penetration depth of $\mathrm{x}$-rays is c.a. $10 \mu \mathrm{m}$, the RXS signal contains information about the ordering processes in this relatively thin layer on the top of the sample that may easily be modified by mechanical treatment.

\section{Discussion}

We have shown that the occurrence (or not) of a step in the intensity of the electronic ordering reflections at $T_{\mathrm{V}}$ is an intrinsic effect not due to deficiencies in stoichiometry, sample quality, etc. Presently we have two different views of understanding our results. (i) An unidentified contamination of the surface is naturally and spontaneously occurring that

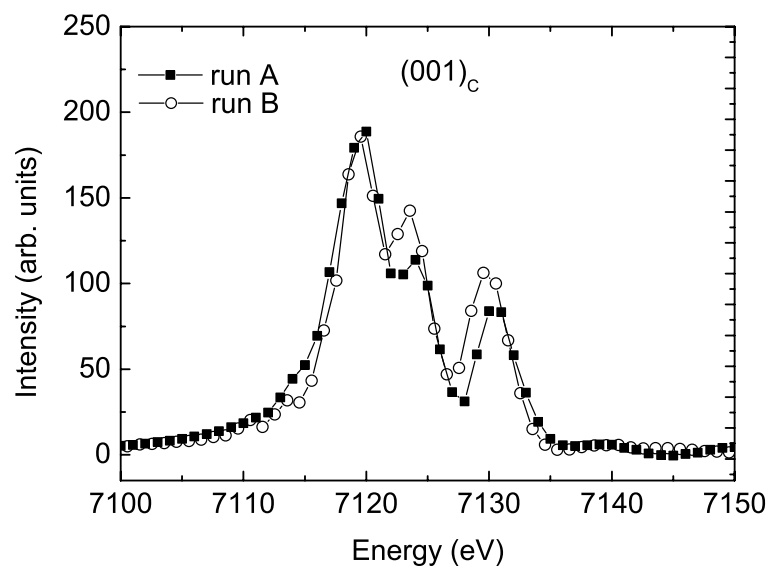

Figure 6. Absorption corrected energy scans of the $(001)_{C}$ reflection in the close neighborhood of the Fe K-edge at $50 \mathrm{~K}$. Both scans have been performed on the same stoichiometric sample and the intensities rescaled for comparison purposes. Open circles correspond to run A (polished well before the experiment) and closed circles represent run B (polishing right before the experiment). The solid lines are guides to the eye.

affects the electronic orders alone. This sort of pollution would develop in the period elapsed between the two experiments, runs $\mathrm{A}$ and $\mathrm{B}$. It is well known that maghemite (or $\gamma-\mathrm{Fe}_{2} \mathrm{O}_{3}$ ) can be formed at the surface of magnetite through the oxidation process, $\mathrm{Fe}^{2+} \rightarrow \mathrm{Fe}^{3+}$. Maghemite has the same structure as magnetite, inverse spinel, and it is ferrimagnetic but it does not undergo a Verwey transition. This oxidation should be accompanied by a contraction of the cubic unit cell $\left(a_{\text {magnetite }}=8.396 \AA \rightarrow a_{\text {maghemite }}=\right.$ $8.3515 \AA$ ) and by the occurrence of internal strains that may propagate well into the magnetite crystal $[19,20]$. This mechanism qualitatively agrees with our overall findings, and the maghemite layer may cause a progressive disruption in the continuity of the charge order to become null at the surface where all $\mathrm{Fe}$ are $\mathrm{Fe}^{3+}$. Diffraction experiments at the $\mathrm{Fe}$ $\mathrm{L}_{2,3}$-edges have shown that an identified dead layer of the order of $1000 \AA$ develops in the sample, and energy scans are very sensitive to this feature [17]. The magnitude of this dead layer is compatible with an oxidation induced maghemite that takes place for months at room temperature. However this oxidation cannot account for the micron deep dead layer found in our experiments at the K-edge. The fact that such a thick dead layer has been found in samples that were polished right before the $\mathrm{x}$-ray experiment precludes the development of such an oxide layer. Therefore the formation of maghemite at the surface seems not to be the key element that accounts for the different features observed in our diffraction experiments.

(ii) As observed in the experiments conducted in this paper (in both runs A and B) a sizable dead layer is observed in the energy scans (figure 1). It is highly probable that the dead layer, made up of powdered $\mathrm{Fe}_{3} \mathrm{O}_{4}$ (see figure 1), is produced by the stresses induced by the mechanical process itself. Hence if the mechanical polishing destroys the surface of $\mathrm{Fe}_{3} \mathrm{O}_{4}$ it should not be at all surprising that the core of the sample under the dead layer has been subtlety affected by this procedure and strains develop and propagate over micron 
size distances beneath the surface. The energy associated should be rather small and hence once the sample is brought back to room temperature and stored for the next experiment strains relax and end up disappearing. This seems to be a very plausible hypothesis.

Strains, whether they are related to process (i) or (ii) or to others, are known to modify the electronic properties of materials, as it has been revealed in experiments e.g. on thin films. As our x-ray experiments probe a relatively thin layer beneath the surface, $\approx 10 \mu \mathrm{m}$, we can consider in a first approximation that our sample behaves as a thin film (thick for the current standards) laterally strained sample. Whether or not this strain hypothesis explains the differences between runs $\mathrm{A}$ and $\mathrm{B}$ in the present $\mathrm{X}$-ray diffraction experiments is still under investigation. The jump that many quantities (magnetization, diffracted intensities of the average high temperature structure, etc) experiences right at $T_{\mathrm{V}}$ is a manifestation of the nucleation and growth of fluctuating domains above $T_{\mathrm{V}}$. Interestingly it is widely accepted that the Verwey transition temperature in magnetite is a good indicator of the quality of the sample: the higher $T_{\mathrm{V}}$ is the better (strain free and stoichiometric) the sample is. In this work we have seen that $T_{\mathrm{V}}$ is identical for both runs, as measured by the same experimental equipment some days apart, in high quality magnetite samples. It is only the mesoscopic features of the electronic orderings that are affected by the different type of runs, and not the purely microscopic features such as the occurrence of charge ordering itself, the magnitude of $T_{\mathrm{V}}$, etc.

The influence of sample preparation and of strains originating at the surface of the sample in relation with phase transitions was a well debated problem in the nineties in compounds such as $\mathrm{SrTiO}_{3}, \mathrm{RbCaF}_{3}, \mathrm{KMnF}_{3}, \mathrm{Rb}_{2} \mathrm{ZnCl}_{4}$, $\mathrm{UO}_{2}, \mathrm{NpAs}, \mathrm{Tb}, \mathrm{Ho}$, etc within the framework of the topic two length scales at the phase transition [21-28]. A detail analysis of the lineshape of the critical scattering in the cubic-to-tetragonal phase transition in $\mathrm{RbCaF}_{3}$ showed that there are 2 different components: a narrow and a broad components that both become critical at the same temperature albeit with different temperature dependences [24]. Since this seminal discovery a whole lot of compounds and phase transitions have been identified as having of two length scales at the phase transition and nowadays it is a well accepted fact. It has been shown that the narrow component arises from a region called the skin of the sample and there is a general consensus that strain fields play a fundamental role at explaining its origin. Despite substantial amount of work, theories are not yet consistent with all the experimental data [29]. Furthermore, it has been shown that mechanically roughened surfaces display this narrow component whereas in smooth surfaces, the narrow component is vanishingly small. The occurrence of two length scales in the fluctuations at the Verwey transition in magnetite will be developed further in a forthcoming publication.

\section{Conclusions}

With respect to the questions raised by our previous paper [13], the rebuttal from Garcia and coworkers [14] and other

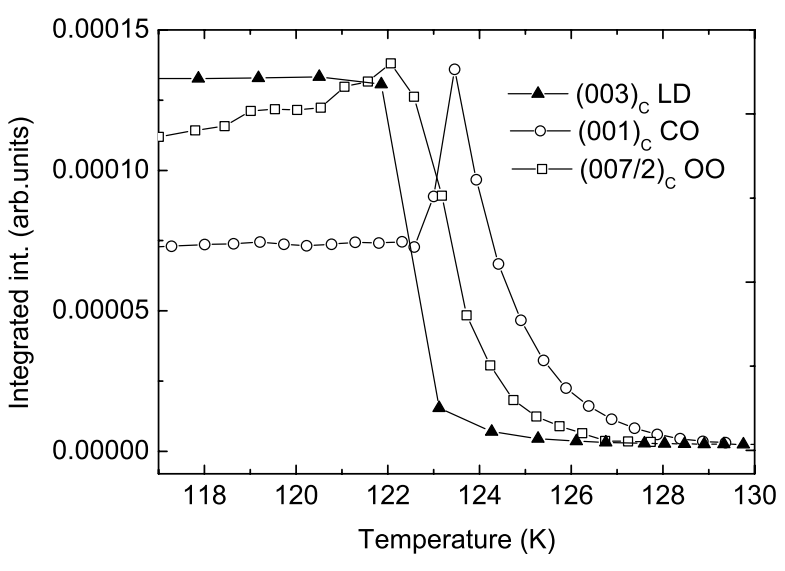

Figure 7. Temperature dependence of the $(003)_{\mathrm{C}},(001)_{\mathrm{C}}$ and $(007 / 2)_{\mathrm{C}}$ peaks intensities for $\mathrm{Fe}_{3} \mathrm{O}_{4}$ polished well before the measurements (run A). The data are the same as in figure 3 but presented in a linear scale. The error bars are of the size of the data points and the solid lines are guides to the eye.

RXS publications on similar matters $[8,11,12,17]$ the situation is as follows.

(i) The occurrence of a dead layer has been thoroughly studied by Chang et al [17] in the context of the analysis of the energy dependence of the $\left(\begin{array}{lll}0 & 0 & \frac{1}{2}\end{array}\right)_{C}$ diffraction peak shown in previous soft RXS experiments $[8,11,12$, 14]. The conclusion of that work is that large spectral differences have been observed due to the occurrence of a dead layer, which is more or less present in most of the samples used in the published data. Although nothing is said on the origin of this dead layer, it seems to be very small in in vacuo cleaved samples or in thin films. A dead layer is always present in our experiments (see figure 1), independently of the run type. This is easily seen in the jump of the intensity above the resonance edge, $\left(\Delta \mu^{-1} / \mu^{-1}\right)_{\text {meas }} \approx 50$, much larger than expected, $\left(\Delta \mu^{-1} / \mu^{-1}\right)_{\text {theory }} \approx 7$ (see figure 1$)$. Despite all efforts we cannot get rid of it by polishing. In view of all the accumulated work, by us and by others, we have to conclude that mechanically polishing or other aggressive treatment of the surface is at the origin of this dead layer. Admittedly the general occurrence of a dead layer at the surface of magnetite needs to be cross checked with other methods of capping the crystal, like chemical etching, plasma etching, etc.

(ii) Regarding the temperature dependence of the intensity of the electronic orders and of the lattice distortions, the run type A data reproduce very nicely the previous findings [13], absence of jump at $T_{\mathrm{V}}$ of the electronic orderings and jump by nearly 2 orders of magnitude within $0.1 \mathrm{~K}$ of $T_{\mathrm{V}}$ of the lattice distortion peak. The absence of jump in the $\left(\begin{array}{lll}0 & 0 & \frac{1}{2}\end{array}\right)_{\mathrm{C}}$ (and related half integer reflections $)$ and in the $\left(\begin{array}{lll}0 & 0 & 1\end{array}\right)_{\mathrm{C}}$ reflections have been shown in previous soft RXS studies on either bulk or thin films magnetite $[8,12,14]$ but went unnoticed to these authors. In figure 7 we have replotted data from figure 3 to show how similar our results are to those in [14], albeit 
with far more points and covering a larger temperature region.

(iii) There is not a phase transition at $T \approx 130 \mathrm{~K}$ in much the same way as there is no phase transition associated to occurrence of the isotropy point in the magnetization and the further spin reorientation along the $(001)_{C}$ direction observed in magnetite. From our past [13] and the present data (figures 3(a) and 4) a saturation of the intensity and a cross over in the correlation length of fluctuations is observed. In addition the location of this temperature is sample dependent: in [13] this is located at around $130 \mathrm{~K}$ whereas in the present sample it is $133 \mathrm{~K}$. Again this is consistent with the spread of temperatures reported in the literature for the isotropy point [30]. Diffraction data in figure 3 of [14] are very sparse, do not cover this temperature range and therefore little can be pulled off on this issue from their work. Concluding on this point, it seems that structural as well as electronic fluctuations do see a change in the fluctuation regime upon the passage through this temperature which, in view of the magnetization results, we have tentatively ascribed to a another kind of manifestation of the vanishing magnetocrystalline anisotropy at the isotropy point. The conclusions drawn in our previous paper [13] hold true.

Summarizing, in this paper we have hypothesized that mechanically polishing the surface of magnetite (i) destroys the sample and creates a dead layer and (ii) induces strains beneath the dead layer that affects mainly the onset of the electronic orders, charge and orbital, by favoring an immediate long range order of these correlations right below $T_{\mathrm{V}}$ (run type B in figure 3 ). The same experiment carried out typically 'a couple of months' later show the electronic orders below $T_{\mathrm{V}}$ occur through a smooth phase transition (run type A in figure 3). Identical behavior has been found in doped and non-stoichiometric samples (figure 5) undergoing a first order phase transition. In order to explain the differences between run type $\mathrm{A}$ and $\mathrm{B}$ we have introduced a second hypothesis that these strains relax in lapse of time of typically a 'couple of months' (although this cannot be determined in any detail) at room temperature.

This smooth behavior at $T_{\mathrm{V}}$ of the electronic orderings is also apparent from figure 4 of [8], from figure 3 of [12] and from figure 3 of [14] and is not what is expected for a very strong first order phase transition as in the case of magnetite. It is important to emphasize that this type of strains do not affect the onset of the lattice distortions as the corresponding reflections invariable show a jump at $T_{\mathrm{V}}$.

The two hypothesis introduced to justify our results are very plausible and the role of strains at the phase transition has been emphasized in a number of compounds having quite different types of phase transitions. We are inclined to think that run type A represents the actual state of bulk magnetite in the absence of strains. The smooth dependence of the electronic component of the Verwey transition is a result that is at the variance of what is accepted for this phase transition and that gives some new openings to the understanding of this fascinating compound.

\section{Acknowledgment}

We want to thank S Grenier for a critical reading of the manuscript and for helpful discussions.

\section{References}

[1] Imada M, Fujimori A and Tokura Y 1998 Rev. Mod. Phys. 701039

[2] Walz F 2002 J. Phys.: Condens. Matter 14285

[3] Garcia J and Subias G 2004 J. Phys.: Condens. Matter 16145

[4] Verwey E J W 1939 Nature 144327

[5] Nazarenko E, Lorenzo J E, Joly Y, Hodeau J L, Mannix D and Marin C 2006 Phys. Rev. Lett. 97056403

[6] Uzu H and Tanaka A 2008 J. Phys. Soc. Japan 77074711

[7] Leonov I, Yaresko A N, Antonov V N, Korotin M A and Anisimov V I 2004 Phys. Rev. Lett. 93146404

[8] Huang D J et al 2006 Phys. Rev. Lett. 96096401

[9] Jeng H T, Guo G Y and Huang D J 2006 Phys. Rev. B 74195115

[10] Zhou F and Ceder G 2010 Phys. Rev. B 81205113

[11] Schlappa J et al 2008 Phys. Rev. Lett. 100026406

[12] Wilkins S B, Di Matteo S, Beale T A W, Joly Y, Mazzoli C, Hatton P D, Bencok P, Yakhou F and Brabers V A M 2009 Phys. Rev. B 79201102

[13] Lorenzo J E, Mazzoli C, Jaouen N, Detlefs C, Mannix D, Grenier S, Joly Y and Marin C 2008 Phys. Rev. Lett. 101226401

[14] Garcia J, Subias G, Herrero-Martin J, Blasco J, Cuartero V, Sánchez M C, Mazzoli C and Yakhou F 2009 Phys. Rev. Lett. 102176405

[15] Paolasini L et al 2007 J. Synchrotron Radiat. 14 301-12

[16] Bland S R, Detlefs B, Wilkins S B, Beale T A W, Mazzoli C, Joly Y, Hatton P D, Lorenzo J E and Brabers V A M 2009 J. Phys.: Condens. Matter 21485601

[17] Chang C F et al 2011 Phys. Rev. B 83073105

[18] Kakol Z and Kozłowski A 2000 Solid State Sci. 2 737-46

[19] Sidhua P, Gilkesa R and Posnera A 1977 J. Inorg. Nucl. Chem. 39 1953-8

[20] Cui Y, Verosub K L and Roberts A P 1994 Geophys. Res. Lett. $21757-60$

[21] Andrews S R 1986 J. Phys. C: Solid State Phys. 193721

[22] Ryan T W, Nelmes R J, Cowley R A and Gibaud A 1986 Phys. Rev. Lett. 56 2704-7

[23] McMorrow D, Hamaya N, Shimomura S, Fujii Y, Kishimoto S and Iwasaki H 1990 Solid State Commun. 76 443-8

[24] Gibaud A, Ryan T W and Nelmes R J 1987 J. Phys. C: Solid State Phys. 20 3833-61

[25] Watson G M, Gaulin B D, Gibbs D, Thurston T R, Simpson P J, Shapiro S M, Lander G H, Matzke H, Wang S and Dudley M 1996 Phys. Rev. B 53 686-98

[26] Langridge S, Stirling W G, Lander G H and Rebizant J 1994 Phys. Rev. B 49 12022-9

[27] Gehring P M, Hirota K, Majkrzak C F and Shirane G 1993 Phys. Rev. Lett. 71 1087-90

[28] Thurston T R, Helgesen G, Gibbs D, Hill J P, Gaulin B D and Shirane G 1993 Phys. Rev. Lett. 70 3151-4

[29] Cowley R A and Shapiro S M 2006 J. Phys. Soc. Japan 75111001

[30] Muxworthy A and McClelland E 2000 Geophys. J. Int. 140 101-14 